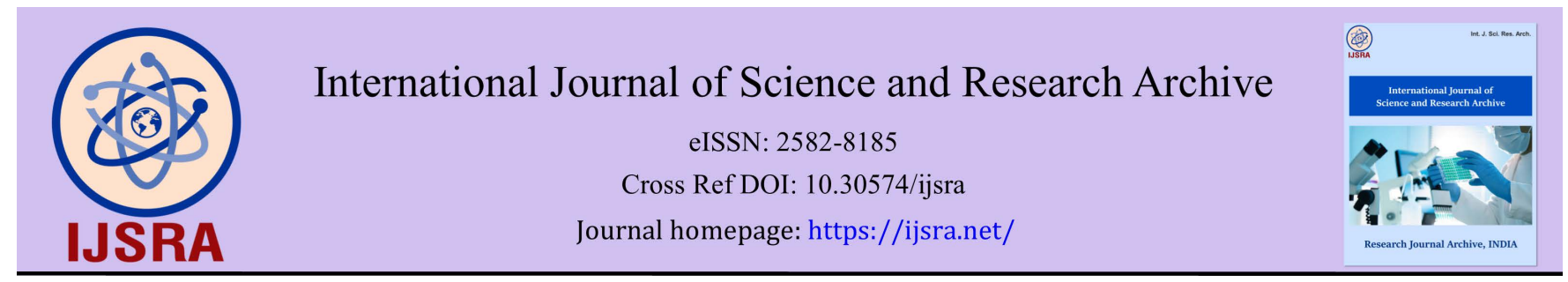

(CASE REPORT)

\title{
Removal of a bullet lying between carotid arteries, following a gunshot injury
}

Athina Zarachi 1, *, Ioannis Komnos 1, Victoria Tsoumani 1, Artemis Andrianopoulou 2, Christina Naka 2, Angelos Liontos ${ }^{3}$, Ioannis Kastanioudakis ${ }^{1}$ and Dimitrios Chatzis ${ }^{4}$

1 Department of Otorhinolaryngology, Head and Neck Surgery, Faculty of Medicine, School of Health Sciences, University of Ioannina, Ioannina, Greece.

${ }^{2}$ Faculty of Medicine, Department of Radiology, School of Health Sciences, University of Ioannina, Ioannina, Greece.

${ }^{3}$ Department of Internal Medicine, Faculty of Medicine, School of Health Sciences, University of Ioannina, Ioannina, Greece.

${ }^{4}$ Vascular Unit, Department of Surgery, University of Ioannina and School of Medicine, University of Ioannina, 45110, Ioannina, Greece.

International Journal of Science and Research Archive, 2021, 03(02), 146-150

Publication history: Received on 02 September 2021; revised on 07 October 2021; accepted on 09 October 2021

Article DOI: https://doi.org/10.30574/ijsra.2021.3.2.0153

\begin{abstract}
Gunshot injuries can be very threatening to the patient's life. A bullet in the neck area after a gunshot usually causes tissue damage and bleeding because of the presence of vital structures in this region. We present the case of a young man that arrived emergently to our hospital because of gunshot injury in the right neck area and the right shoulder. He was hemodynamically stable, with no laryngeal edema or hematoma. The cervical radiography showed a foreign body lying on the right side of the spine, in front of the third cervical vertebra. The CT scanning revealed a metallic foreign body, lying between the internal carotid artery and the external carotid artery, without causing bleeding in the surrounding tissues. A bullet was also found in the right shoulder area. A barium esophagography showed no contrast agent escape. An emergency operation was performed, under general anesthesia. The metallic bullet was found under an enlarged submandibular lymph node and was removed. The bullet removal caused vessel intraoperative bleeding, that was repaired satisfactory. No postoperative complications were noticed and patient was discharged home.
\end{abstract}

Keywords: Bullet; Gunshot injury; Removal; Neck area; Carotid artery

\section{Introduction}

Gunshot wounds are not the commonest, but leading cause of injury, following automobile accidents and falls. A bullet can be found everywhere in the human body and usual entry points are: the head, the neck, the spine and the chest. The severity of injury resulting from gunshot wounds varies according to the weapon used, the distance from which the patient is shot as well as the entry point of injury and the type of injury (perforating, hemorrhagic etc). They can cause functional and aesthetic consequences for the patient, even death. In order to achieve an early management of these patients, doctors must firstly focus on the status of the airway, breathing and circulation. A neck area gunshot can cause bleeding from the injury, while the subsequent hematoma probably causes swelling that can compromise the airway. A laryngeal edema can also be caused after neck injury. Control with either an endotracheal tube or tracheotomy should be considered early. The hemodynamic patient status should be controlled. Gunshot injuries to the neck area are associated with high morbidity and mortality due to the complex anatomy and presence of various vital structures in this region[1-10].

\footnotetext{
${ }^{*}$ Corresponding author: Athina Zarachi

Department of Otorhinolaryngology, Head and Neck Surgery, Faculty of Medicine, School of Health Sciences, University of Ioannina, Ioannina, Greece.
}

Copyright $(2021$ Author(s) retain the copyright of this article. This article is published under the terms of the Creative Commons Attribution Liscense 4.0. 
We present a case of gunshot injury to the neck area in which the bullet's trajectory passes through the neck region and especially between the carotid arteries, without damaging any vital structures.

\section{Case report}

A 31-year-old young man addressed the emergency department of our hospital because of gunshot injury, five hours before the arrival time. He presented with perforating injury in the right neck area and a right shoulder injury. Through clinical examination, the patient was hemodynamically stable and fully conscious. He did not appear laryngeal edema, hematoma or airway injury. Anteroposterior and lateral cervical radiography showed a foreign body lying on the right side of the neck area, next to the spine and in front of the third cervical vertebra (Figure 1,2). The patient underwent a computed tomography (CT) of the head and neck, the spine and the chest. The CT scanning revealed a metallic foreign body, lying between the internal carotid artery (ICA) and the external carotid artery (ECA). The metallic foreign body caused strong artifacts that changed the CT image, so the exact damage was not clarified. The bullet was lying between carotid arteries, without causing bleeding in the surrounding tissues (Figure 3). A metallic foreign body was also found in the right shoulder area. A barium esophagography showed normal primary peristalsis with no contrast agent escape.

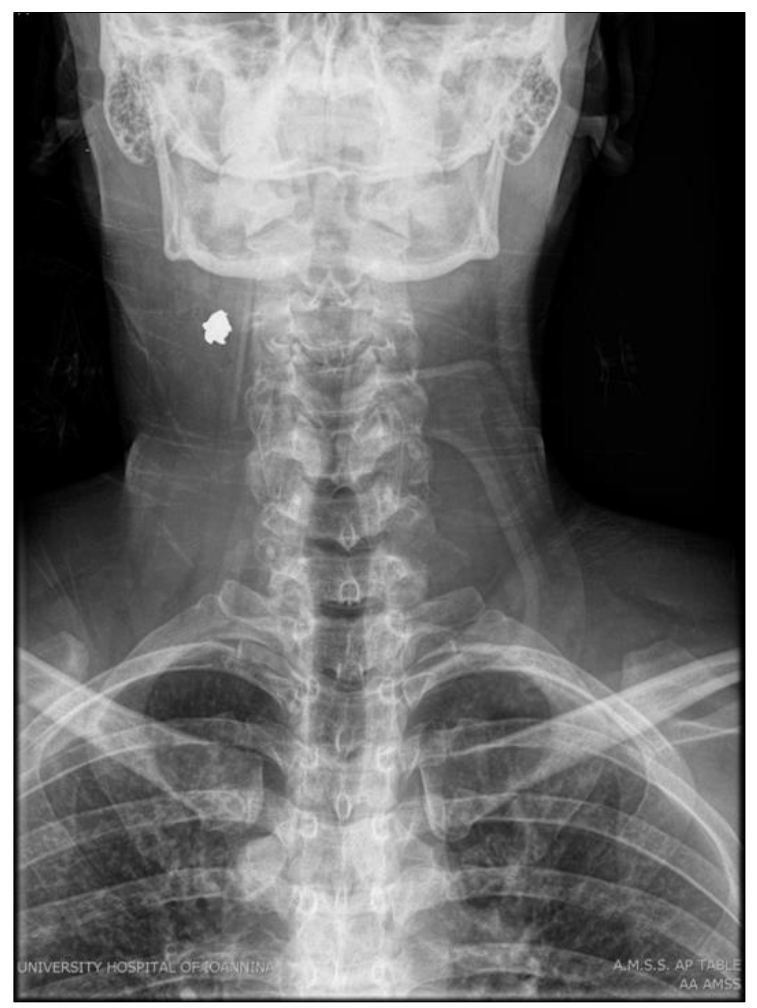

Figure 1 Anteroposterior cervical radiography shows a foreign body on the right side of the neck, next to the spine

An emergency operation was performed, under general anesthesia. A surgical incision was held on the right neck area along the anterior lip of sternocleidomastoid muscle. Sequential dissection of the cervical layers included skin, supraplatysmal fat, platysma muscle, and subplatysmal fat. The metallic bullet was found under an enlarged submandibular lymph node and was removed. Its length was about one centimeter and its surface was sharp and uneven (Figure 4). 


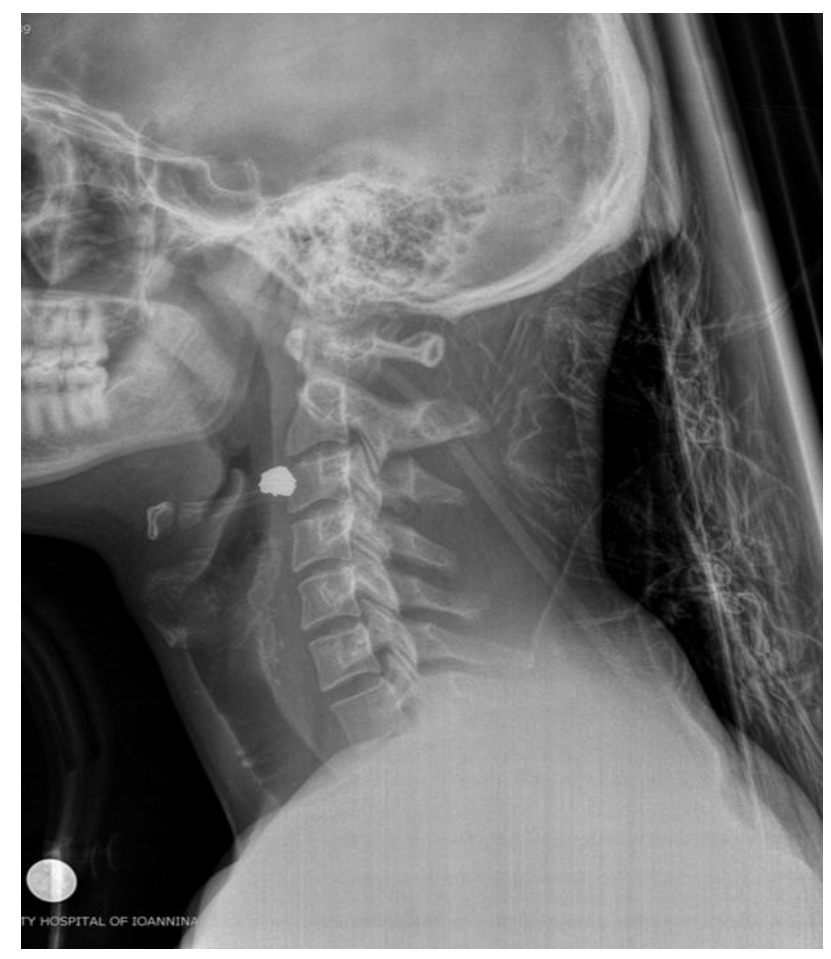

Figure 2 Lateral cervical radiography shows a foreign body in front of the third cervical vertebra

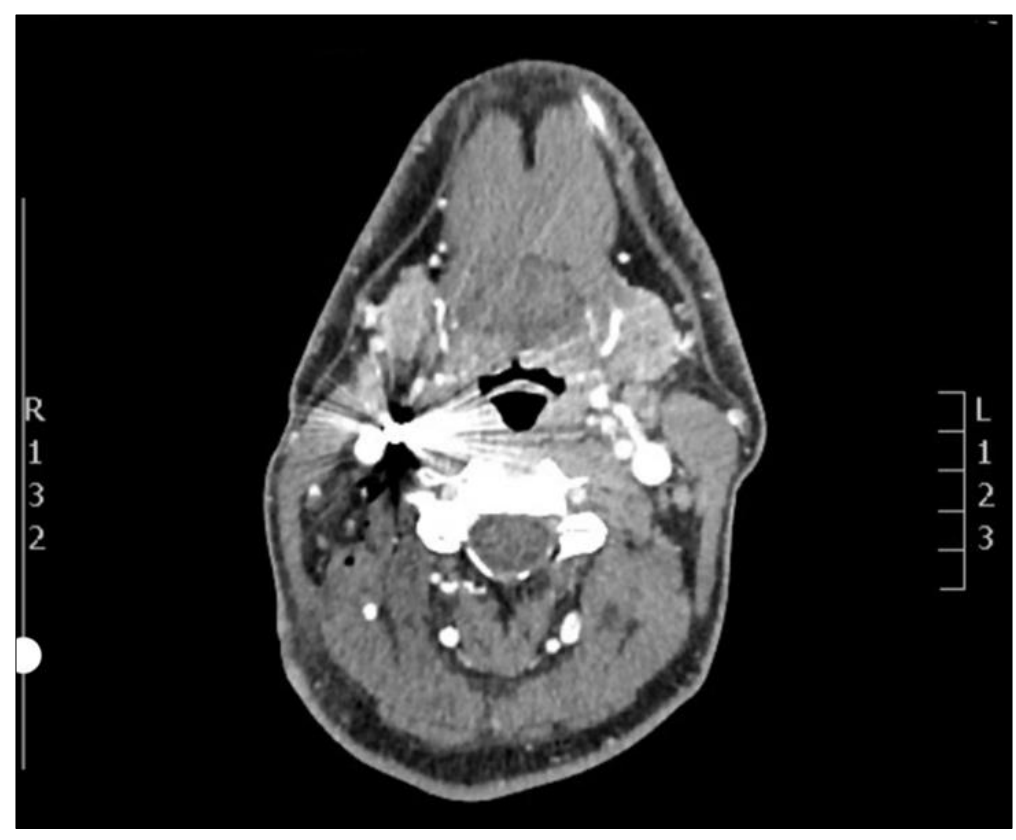

Figure 3 CT shows a metallic foreign body, in the right neck area, lying between the internal carotid artery and the external carotid artery. The metallic foreign body causes strong artifacts

An intraoperative bleeding caused during the removal of the bullet, probably because of the sharp surface of the bullet. Ligation was used in order to repair the bleeding vessels. Wound was closed in layers by approaching the tissues and using sutures. No postoperative complications were noticed and patient was discharged with absolutely satisfactory condition from the ward on fifth postoperative day. 


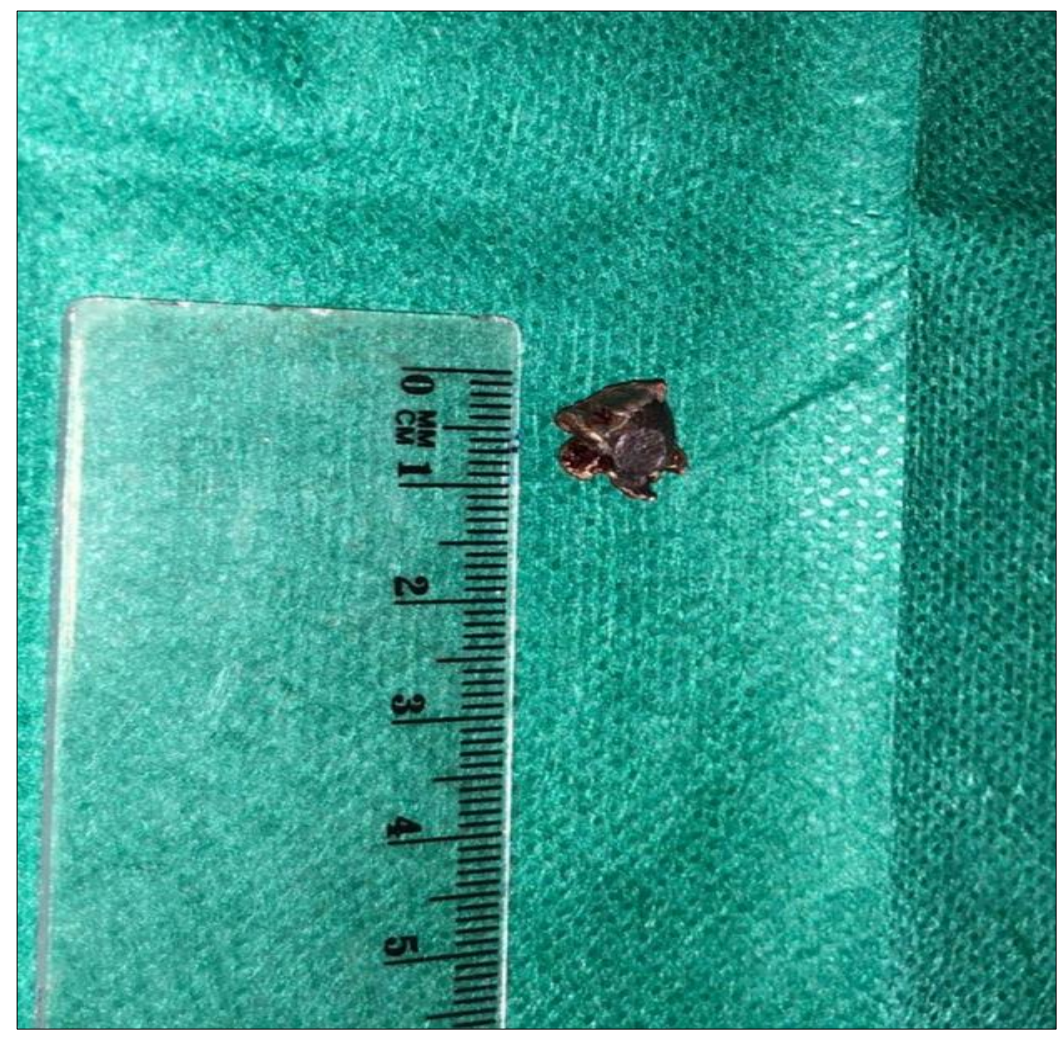

Figure 4 The bullet length was about one centimeter.

\section{Discussion}

Gunshot wounds are associated with a high mortality and frequently require operative intervention. The diagnostic and therapeutic challenges of these injuries vary and depend on the entry point and structures of injury. The evaluation and management of penetrating trauma to the neck is a controversial topic. The zone of head and neck region that is injured plays an important role. The zone I is the area below the lower boarder of cricoid cartilage, the zone II is between the lower boarder of cricoid cartilage below and up to the angles of mandible, while zone III is the area from the angles of the mandible up to the skull base. Through the mechanism of shock waves that produced after injury, it is possible to be caused vessel thrombosis, embolism or pseudoaneurysm. In the past, there has been a trend toward less invasive diagnostic and therapeutic techniques. Contemporary protocols propose the immediate surgical exploration when patients are unstable, such as those with active bleeding or expanding hematoma. Stable patients with zone I or III injuries are usually evaluated by angiography due to limited surgical access to vascular structures. Zone II injuries, remain the most controversial. Authors propose as initial assessment the immediate exploration without angiography, the exploration based on angiographic findings or the clinical evaluation alone. Indications for immediate surgical exploration of zone II neck injuries are the presence of active external hemmorhage, hemoptysis, stridor, hypovolemic shock, obvious esophageal injury, obvious tracheal injury or air bubbling from the wound [11-16].

\section{Conclusion}

Penetrating neck trauma needs a careful management that depends on the cause of injury, the extent of damage, the appearance of complications and indications for immediate surgical exploration or not. We report the case of a young man that was presented to our hospital emergently, because of a gunshot. We were called to face the zone II neck trauma and remove the bullet embedded. Although gunshots in this specific neck area usually cause bleeding due to the vessels injury, in our case the bullet was lying between the ICA and the ECA, without causing bleeding. Despite the fact that the patient did not appear indications for immediate surgical exploration, we proceeded to it. The artifacts on CT caused by the metallic foreign body that made the damage evaluation not clear, as well as the position of the bullet, in between ICA and ECA, were the strongest reasons to explore the damage immediately through a surgical operation. 


\section{Compliance with ethical standards}

\section{Acknowledgments}

There are no acknowledgments.

\section{Disclosure of conflict of interest}

The authors declare no conflicts of interest regarding the publication of this paper.

\section{Statement of informed consent}

Informed consent was obtained from all individual participants included in the study.

\section{References}

[1] Nicol JW, Yardley MPJ, Parker AJ. Pharyngolaryngeal migration: a delayed complication of an impacted bullet in the neck. The Journal of Laryngology \& Otology. 1992; 106(12): 1091-1093.

[2] Maguire D, Lodewyks C, Yamashita MH. Lost but eventually found: delayed removal of an intramyocardial bullet. Canadian Journal of Anesthesia/Journal Canadien D'anesthésie. 2020; 68(2): 256-257.

[3] Sugita K, Doi T, Sato O, Takaoka Y, Mutsuga N, Tsugane R. Successful Removal of Intracranial Air-Gun Bullet With Stereotaxic Apparatus. Journal of Neurosurgery. 1969; 30(2): 177-181.

[4] Mineo RC, Gittins ME. Arthroscopic removal of a bullet embedded in the acetabulum. Arthroscopy: The Journal of Arthroscopic \& Related Surgery. 2003; 19(9): e121-e124.

[5] Cantrell C, Gerlach E, Butler B, Sheth U, Tjong V. The role of arthroscopy in bullet removal: A systematic review of the literature. Journal of Orthopaedics. 2020; 22: 442-448.

[6] Choh JH, Adler RH. Penetrating bullet wound of chest with bronchoscopic removal of bullet. The Journal of Thoracic and Cardiovascular Surgery. 1981; 82(1): 150-153.

[7] WATERS RL, ADKINS RH. The Effects of Removal of Bullet Fragments Retained in the Spinal Canal. SPINE. 1991; 16(8): 934-939.

[8] Hollier L, Grantcharova EP, Kattash M. Facial gunshot wounds: A 4-year experience. Journal of Oral and Maxillofacial Surgery. 2001; 59(3): 277-282.

[9] Godhi S, Mittal GS, Kukreja P. Gunshot Injury in the Neck With an Atypical Bullet Trajectory. Journal of Maxillofacial and Oral Surgery. 2010; 10(1): 80-84.

[10] Holt GR, Kostohryz G. Wound Ballistics of Gunshot Injuries to the Head and Neck. Archives of Otolaryngology Head and Neck Surgery. 1983; 109(5), 313-318.

[11] Wang CHJ, Huang H, Yang K, et al. Patho-mechanism of shock wave injuries on femoral artery, vein, and nerve: an experimental study in dogs. Injury. 2002; 33: 439-446.

[12] Doctor VS, Farwell DG. Gunshot wounds to the head and neck. Current Opinion in Otolaryngology \& Head and Neck Surgery. 2007; 15(4): 213-218.

[13] Monson DO, Saletta JD, Freeark RJ. Carotid Vertebral Trauma. The Journal of Trauma: Injury, Infection, and Critical Care. 1969; 9(12): 987-999.

[14] Demetriades D. Penetrating Injuries of the Neck in Patients in Stable Condition. Archives of Surgery. 1995; 130(9): 971.

[15] Sofianos C, Degiannis E, Van den Aardweg MS, Levy RD, Naidu M, Saadia R. Selective surgical management of zone II gunshot injuries of the neck: A prospective study. Surgery. 1996; 120(5): 785-788.

[16] Ghnnam WM, Al-Mastour AS, Bazeed MF. Penetrating Neck Trauma in a Level II Trauma Hospital, Saudi Arabia. ISRN Emergency Medicine. 2012; 1-6. 